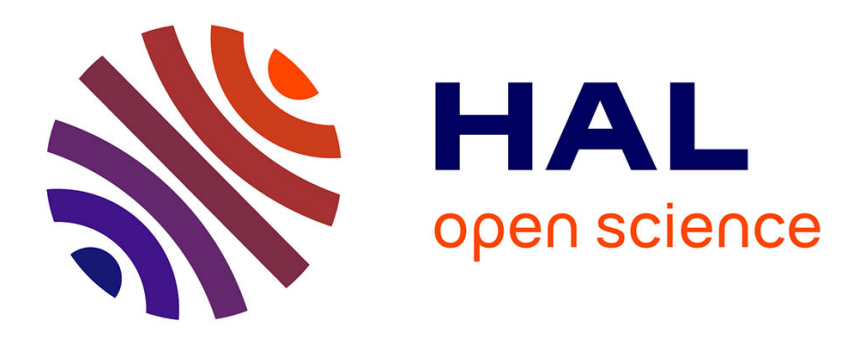

\title{
Le diagnostic ou "La douleur révélatrice " Chez Abû Bakr al-Râzî
}

Okba Djenane

\section{To cite this version:}

Okba Djenane. Le diagnostic ou " La douleur révélatrice " Chez Abû Bakr al-Râzî. 2009. hal00615308

\section{HAL Id: hal-00615308 \\ https://hal.science/hal-00615308}

Preprint submitted on 18 Aug 2011

HAL is a multi-disciplinary open access archive for the deposit and dissemination of scientific research documents, whether they are published or not. The documents may come from teaching and research institutions in France or abroad, or from public or private research centers.
L'archive ouverte pluridisciplinaire HAL, est destinée au dépôt et à la diffusion de documents scientifiques de niveau recherche, publiés ou non, émanant des établissements d'enseignement et de recherche français ou étrangers, des laboratoires publics ou privés. 


\title{
Le diagnostic ou « La douleur révélatrice »
}

\author{
Chez Abū Bakr al-Rāzī
}

\section{Okba DJENANE}

\section{Résumé :}

Le diagnostic provisoire, chez al-Rāzī, exige la connaissance de la maladie par l'observation, où la douleur demeure une révélation de la défaillance fonctionnelle de l'organisme. C'est pourquoi, il accorde une place importante aux malades et aux expressions de leur mal, comme signes essentiels pour le diagnostic et pour la différenciation entre la maladie et la douleur. Puis, il passe au pronostic ou «le diagnostic déguisé ». Un préjugé sur la maladie et son terme devient nécessaire quand la supériorité philosophique du diagnostic n'arrive pas à la supériorité pragmatique voulue.

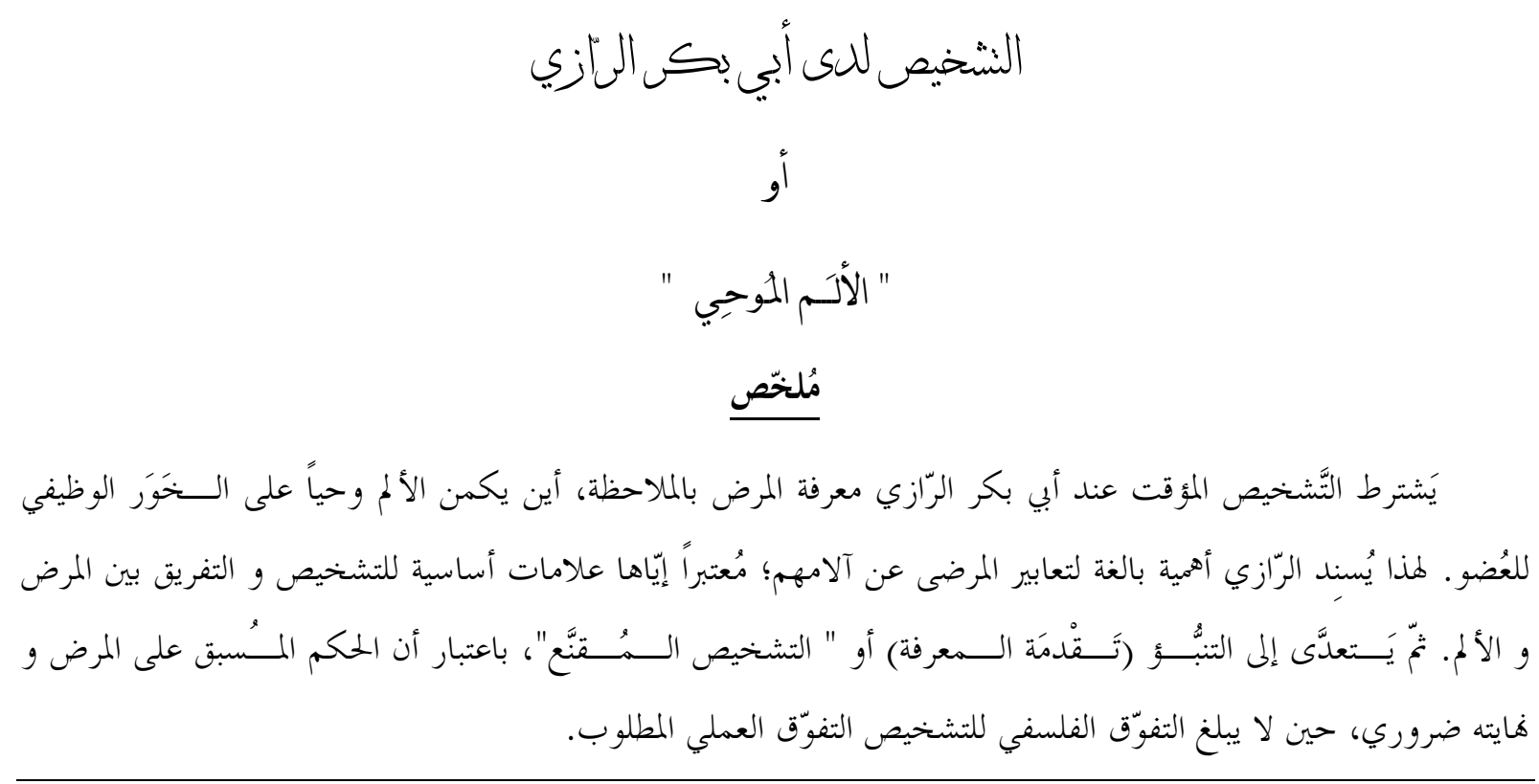


«(..) Quant à la rougeole, plusieurs symptômes qui lui sont communs avec la petite vérole: la chaleur est très grande, et la poitrine éprouve un sentiment considérable de resserrement, surtout s'il $y$ a toux, avec démangeaison des oreilles et du nez. ».

Al-Rāzī, al-Ğidrī wa-l-ḥaṣbah, p. 7.

Pour pouvoir vaincre la maladie, le médecin doit identifier la maladie ou savoir faire le diagnostic (al-ta'rïf). C'est-à-dire, reconnaître la maladie du point vue anatomique, physiologique et reconnaître son espèce nosologique ${ }^{1}$, aussi les signes (al-'alāmāt) qui la désignent, et percevoir les causes (al-asbāb) qui se cachent derrière ces signes. En outre, les signes des maladies et leurs traitements sont classés en fonction des observations habituelles sur les humeurs en cause ; le diagnostic se fait par la différenciation de leur excès ou de leur défaut, par la présence des différents éléments et leurs qualités : froid, chaud, sec et humide. Dans al-Hâầi par exemple, al-Rāzī décrit le déséquilibre entre chaud et froid ainsi : «Signes d'une corruption de la complexion du foie due à la chaleur : intensité de la soif, faiblesse de l'appétit. Signes du froid: intensité de l'appétit, blancheur des lèvres et de la langue, peu de rougeur, soif, blancheur de l'urine ${ }^{2}$.».

Dans son diagnostic, al-Rāzī accorde une place importante à la douleur, notamment dans les cas cliniques qu'il décrit et qui révèlent non seulement un aspect théorique, mais aussi des essais cliniques et pratiques très élaborées, du fait qu'il corrige Galien (131 ? 201 ? apr. J.-C.), soit sur le plan logique, soit sur le plan clinique ${ }^{3}$. Il rapporte dans son livre : Les Doutes sur Galien, des expériences cliniques et dénombre des maladies inconnues de Galien, comme lors de ses diagnostics sur les ulcères d'estomac, qu'il juge inexacts ${ }^{4}$. Ou, encore, les cas de fièvres, décrites à l'hôpital de Bagdad, qui ne sont pas semblables à la description de Galien. De plus, il lutte contre le diagnostic basé sur l'examen des urines, sans visiter le malade ${ }^{5}$.

1. GRMEK, M. Dražen. «La pratique médicale». In: Hippocrate de Cos. De l'art médical. Danielle GOUREVITCH, Mirko GRMEK et Pierre PELLEGRIN (intro.). Paris : Librairie générale française, cop. 1994. Le Livre de poche. Bibliothèque classique ; 704. pp. 40-59, p. 53.

${ }^{2}$. Al-Rāzī, al-Hāwīi, vol. VII, p. 66-67, cité par Danielle JACQUART. La science médicale occidentale entre deux renaissances : XIIe s.-XVe s. Aldershot ; Brookfield : Variorum, 1997, p. 166.

3. Selon George SARTON, on ne trouve pas chez Galien des rapports cliniques intéressants, il faut attendre alRāzī (la seconde moitié du neuvième siècle.) pour en avoir. Introduction to the History of Science. Baltimore. Maryland Etats-Unis d'Amérique : The Williams and Wilkins Company Baltimore, 1927. pp. 609-610. 5 vol., 839-1251-2155 p, p. 240.

4. Dominique LECOURT (publié sous la dir.). Dictionnaire d'histoire et philosophie des sciences. Paris : PUF, 1999. (41-Vendôme : Impr. des PUF), XXI-1032 p, pp. 801-802.

5 . Arturo CASTIGLIONI. Histoire de la médecine. Juliette BERTRAND et Ferdinand GIDON (traduction par). Edition française revue par les soins de l'auteur. Paris : Payot, 1931, p. 232. 
En outre, dans al-Ḥ̂̄wì al-Rāzī avait motionné un nombre important d'observations cliniques, «concernant les maladies éruptives avec les règles hygiéniques remarquables comme la mise du malade dans une atmosphère tiède. Avec soins prophylactiques soutenus des yeux, de la bouche et des fosses nasales ${ }^{6}$. $»$. Une partie de ces observations sont dues à alRāzī lui-même et une autre partie provient de sa très vaste connaissance de la littérature médicale $^{7}$. Max MEYERHOF a publié 33 observations $^{8}$ de ce genre dues à al-Rāzī, cette étude montre une différence entre une médecine exposée dans les grands traités hérités des Grecs, et une médecine concrète basée sur l'expérience. Voici à titre d'exemple le Cas $\mathrm{n}^{\circ} 12$ (Cas d'obésité et de goutte) :

Parce que Ibn al-Husayn b. 'Abdawayh était obèse, les médecins croyaient qu'il avait un tempérament humide: ils étaient incapables de distinguer entre un homme charnu (lahìm) et un homme gras (al-badan al-šahim). Il avait eu une attaque douloureuse aux articulations qui avait disparu. Je lui fis plusieurs saignées et lui administrai une fois par semaine un laxatif qui élimine la bile jaune, car ce mélange [nocif d'humeurs] était du pus jaune. Je lui prescrivis des aliments sucrés forts et graisseux. La maladie s'atténua et il ne lui arrivait que des choses sans importance. Quand il eut suivi ce traitement pendant un temps assez long, il guérit complètement et son corps commença à perdre sa chair ${ }^{9}$.

Pour avoir un diagnostic moderne, il suffit de remplacer le terme de « bile jaune » par « acide urique ». C'est pourquoi, le Dr. MEYERHOF, qualifie ce traitement de logique ${ }^{10}$.

L'autre exemple, plus significatif, est celui de la variole et de la rougeole, dans lequel alRāzī parle de la maladie à partir de ses observations patientes et minutieuses personnelles. Toutefois, il en parle comme d'une chose connue, et tout ce qu'il dit des symptômes est si insuffisant, qu'il n'y a pas lieu d'en conclure quelque chose pour le diagnostic. A propos de la petite variole, de celle qui est la plus grave, il dénombre ses symptômes annonciateurs : une fièvre rémittente, une « grande douleur au dos, des anxiétés fortes, une chaleur considérable

\footnotetext{
${ }^{6}$. Sleïm AMMAR. En souvenir de la médecine arabe : quelques-uns de ses grands noms. Tunis : Bascone et Muscat, 1965, p. 121.

7. Certains voient les observations cliniques d'al-Rāzī, comme pouvant être comparées à les Epidémies d'Hippocrate, surtout par la structure et la forme du texte. Voir Mirko D. GRMEK (sous la dire.). Histoire de la pensée médicale en Occident. t. I, Antiquité et Moyen âge. Bernardino Fantini (avec la collab. de) ; Maria Laura Bardinet Broso (trad. de). Paris : éd. du Seuil, 1995, p. 138.

8. Les trente-cinq observations cliniques sont insérées dans le septième volume d'al-Hāwī. En revanche, alTanūhī (m. 994 J.-C/ 384 h) évoque un ensemble de cas montrant une excellente méthode qui se base sur l'observation et l'expérimentation et qui révèlent l'attitude d'un philosophe intégralement rationaliste. A titre d'exemple, voir le cas d'un jeune homme qui crachait du sang. Al-Fară̌ ba'da al-šidda [Le soulagement après la rudesse]. 'Abūd AL-ŠĀLĞ̄i (éd.). Beyreuth, Liban : Dār Șādir, 1978, vol. IV, pp. 199-200.

9. Max MEYERHOF."Thirty-three clinical observations by Rhazes (circa 900 A. D.)". In: Penelope Johnstone (Edited by), Studies in medieval Arabic medicine, London: Variorum Reprints, 1984. pp. 321-356. Variorum reprint ; CS204, Cas n ${ }^{\circ} 12$, p. 339.

10. Selon Georges C. ANNAWATI. « La médecine arabe jusqu'au temps d'Avicenne ». Les mardis de dar elsalam. Paris : J. Vrin ; Le Caire : Centre d'études Dar el-Salam, 1965, p. 202.
} 
dans le corps, une couleur très vive et très rouge, et surtout à la gorge. Quant à la rougeole, plusieurs symptômes qui lui sont communs avec la petite vérole : la chaleur est très grande, et la poitrine éprouve un sentiment considérable de resserrement, surtout s'il y a toux, avec démangeaison des oreilles et du nez ${ }^{11}$. ».

Il est certain pour al-Rāzī, que l'art médical est un art qui a pour but la guérison. C'est pourquoi, l'aspect descriptif de la maladie est tout à fait orienté vers le traitement qu'il expose avec beaucoup de détails et de nombreuses observations qui attestent l'intérêt accordé aux malades et aux expressions de leur mal. Cette façon de décrire et de caractériser les douleurs (aigues, chroniques, récurrentes, lancinantes, etc.), ouvre sur une sémiologie notable ${ }^{12}$ et une nosologie remarquable. Cependant le principe nosographique est loin d'être découvert : «Une maladie étant donnée, [il faut] déterminer sa place dans un cadre nosologique. ${ }^{\text {,". } . » . ~}$

Si nous tenons compte de la définition du corps sain chez al-Rāzī : " Qui, dans sa totalité ou une partie de ses organes, accomplit sans douleur les fonctions qui lui son propres, selon ses habitudes courantes ${ }^{14}$, » la douleur demeure un indice capital pour le diagnostic et une révélation de la défaillance fonctionnelle de l'organisme. Elle peut apparaître, soit par un changement qui touche l'organe (comme une fracture), soit par un changement dans le tempérament (il devient froid ou chaud). En outre, l'annulation ou la diminution des fonctions des organes ou de leur douleur sont à l'origine des maladies : «Quand l'œil voit normalement et sans douleur, il est en bonne santé. Mais s'il ne voit rien du tout ou très faiblement, ou avec douleur, c'est qu'il est malade du fait de la présence de cette douleur, même si la vue n'a subi aucune diminution ${ }^{15}$. ». Cet exemple montre qu'al-Rāzī a tendance à utiliser la doctrine de « la douleur-maladie ».

En conclusion, l'intervention médicale chez al-Rāzī, pose, en premier lieu, le diagnostic, à partir d'une bonne connaissance du malade (son tempérament, son régime ordinaire, ses maladies antérieures ou même des maladies héréditaires ${ }^{16}$ ). De plus, il prend en considération

\footnotetext{
11. Al-Rāzī. Kitāb al-Ğidrī wa-l-haṣbah [Livre de la variole et de la rougeole]. Mahmoud NADJMABADI (éd. Critique, trad. et comment.). 3e éd. Téhéran : Presse Universitaire de Téhéran; ${ }^{\circ} 140,1992$, p. 7. [ici K. alGidrī ].

12. Danielle JACQUART; Françoise MICHEAU. La médecine arabe et l'Occident médiéval. Paris: Maisonneuve et Larose, 1996. 1 vol., 271 p, p. 67.

${ }^{13}$. Eugène BOUCHUT. Histoire de la médecine et des doctrines médicales : leçons faites à l'Ecole pratique de la Faculté de médecine en 1862, 1863 et 1864. Paris : Baillière, 1864, p. 252.

${ }^{14}$. Cité dans Anne-Marie MOULIN. « Douleur et médecine dans la tradition arabo-musulmane ». In : Journal International de Bioéthique : Islam - Méditerranée : la perception sociale de la mort et de la douleur. 2001, $1^{\text {ère }}$ Partie, n4, pp. 39-50,

15 . Ibid., p, 44.

16. Voir par exemple le $1^{\text {er }}$ Cas «La Pyonephrosis » d'Abdallah b. Sawāda dans MEYERHOF. "Thirty-three clinical observations", op. cit.
} 
la relation entre le malade et les saisons, dans son diagnostic, et tout ce qui a à voir avec le lien entre le macrocosme et le microcosme ${ }^{17}$.

Le fait d'insister sur la valeur de l'observation clinique objective sans préjugé dogmatique, fait le grand mérite d'al-Rāzī. Néanmoins, notre auteur apparaît moins clair dès qu'il fournit des explications purement théoriques. C'est pourquoi, il est parfois difficile de le comprendre ${ }^{18}$.

Enfin, selon al-Rāzī, il reste certaines maladies graves, qui sont moins difficiles à diagnostiquer que d'autres et qui sont moins dangereuses. En effet, la difficulté du diagnostic résulte de l'interrogatoire difficile du malade, qui ne sait pas exprimer son mal, position qui conduit vers d'autres éléments de diagnostic comme : le toucher, la vue et l'odorat, éléments qui restent primordiaux pour bien écouter un corps, afin de bien diagnostiquer.

L'importance apportée à la douleur, en adoptant la théorie de la douleur-maladie, amène al-Rāzì à différencier entre les maladies, ou bien entre les douleurs. Formuler un diagnostic différentiel (tašh̆iṣ muqāran) c'est nommer le trouble pathologique qui apparaît, en notant la particularité de la maladie, son évolution et les accidents (al-a $\left.{ }^{\top} r \bar{r} d\right)$ passés ou avenir ${ }^{19}$. De plus, il consiste, soit à différencier entre les causes et les signes d'une seule maladie, soit à différencier entre les maladies qui se ressemblent, comme le cas de la variole et la rougeole, dont il ne prend pas en compte la possibilité de contagion en restant totalement dans le cadre de la pathologie humorale ${ }^{20}$. Cependant, il expose avec beaucoup d'authenticité les différences et les signes pronostics de la petite vérole ${ }^{21}$.

En outre, dans Quelle est la différence, livre de diagnostic différentiel par excellence ${ }^{22}$, al-Rāzī fournit les maladies qui ne se ressemblent pas et celles qui se ressemblent, ou les

\footnotetext{
17. René TATON. (sous la dir.). La Science antique et médiévale : des origines à 1450. [Texte imprimé] par Roger ARNALDEZ, Jean BEAUJEU, Guy BEAUJOUAN [et al.]. Paris : PUF, 1994. 1 vol., VIII-627 p. «Quadrige », $\mathrm{n}^{\circ}$ 173. 0291-0489. Histoire générale des sciences ; 1, p. 516.

18. Mohammad H. KĀMIL. «Ṭibb al-Rāzī [La médicine d'al-Rāzī]». In : Al-Rāzī. Kitāb al-Muršid aw al-Fūṣūl [Les Aphorismes]. Egypte : Hağr, 1995, p. 149.

19. Al-Rāzī. Kitāb al-Muršid aw al-Fușūl [Les Aphorismes]. El-Arbi MOUBACHIR (aphorismes présentés et trad. de l'arabe) [le titre donné par l'éditeur est Guide du médecin nomade]. Paul MILLIEZ (Liminaire de). Paris : Sindbad, 1980. 195 p, aph. 368, p. 151.

${ }^{20}$. Dražen M. GRMEK (sous la dir.). Histoire de la pensée médicale en Occident. t. I, Antiquité et Moyen âge. Bernardino Fantini (avec la collab. de) ; Maria Laura Bardinet Broso (trad. de). Paris : éd. du Seuil, 1995. 1 vol., 382 p. Science ouverte, p. 138.

${ }^{21}$. Etienne TOURTELLE. Histoire philosophique de la médecine : depuis son origine jusqu'au commencement $d u$ XVIIIe siècle. Paris : Chez Levrault, Schoell et compagne, 1804, p. 272.

${ }^{22}$. Il expose la raison qui l'a incité à écrire ce livre comme suit : "Quand j'ai constaté que les médecins de notre temps ne savent des maladies que ce qu'ils ont imaginé d'après les livres, de leurs signes et de leurs causes cités et comment ces signes et ces causes peuvent s'associer et les maladies se ressembler [...] j'ai décidé d'écrire un livre des causes, des signes, des maladies, pour que l'on y pense lorsque on les rencontre [...] Son intérêt est grand pour ce qui est de se souvenir de tout ce qui ressemble de se méfier de l'équivoque.». Al-Rāzī,
} 
douleurs similaires pour un ensemble de maladies. En particulier, dans Livre de la colique, alRāzī nous différencie les genres de colique et mentionne les plus fréquents, il nous décrit le troisième genre ainsi : "Le troisième genre est précédé par une fièvre ; la douleur dans ce genre augmente graduellement, et n'atteint pas son paroxysme [nawbah] en une ou deux heures, mais en quelques jours, avec une forte fièvre. Les deux premiers genres ne sont pas accompagnés de fièvre, et si jamais celle-ci apparaît, elle leur est bon remède, et elle serait due aux remèdes chauds ${ }^{23}$. ».

De ce fait, la connaissance de la douleur, avec ses signes et ses causes, résulte de la pratique quotidienne de la médecine et de sa confrontation régulière aux difficultés du diagnostic différentiel et de ce qui résulte en cas d'erreur. Il pratique aussi le diagnostic différentiel par le traitement. Dans son La Colique il donne l'exemple d'avoir donné le médicament à un groupe et pas à un autre atteint par la même maladie. Puis il en analyse les résultats.

K. Mā al-Farq aw al-Furūq [Quelle est la différence]. Salman KATTAYA (éd.), Alep : Ma'had al-Turāt al-'Ilmī, 1978, p. 1.

23. Al-Rāzī. Kitāb al-Qūlunğ [Livre de la colique]. Soubhi M. HAMMAMI (éd. critique et trad. fr.). Alep : Université d'Alep, 1983. 274 p, p. 54 